\title{
A Public Key Encryption Scheme Secure against Key Dependent Chosen Plaintext and Adaptive Chosen Ciphertext Attacks
}

\author{
Jan Camenisch ${ }^{1}$, Nishanth Chandran ${ }^{2}$, and Victor Shoup ${ }^{3}$ \\ ${ }^{1}$ IBM Research, work funded by the European Community's Seventh Framework \\ Programme (FP7/2007-2013) under grant agreement no. 216483 \\ ${ }^{2}$ UCLA, work done while visiting IBM Research \\ ${ }^{3}$ NYU, work done while visiting IBM Research, supported by NSF award number \\ CNS-0716690
}

\begin{abstract}
Recently, at Crypto 2008, Boneh, Halevi, Hamburg, and Ostrovsky (BHHO) solved the long-standing open problem of "circular encryption," by presenting a public key encryption scheme and proving that it is semantically secure against key dependent chosen plaintext attack (KDM-CPA security) under standard assumptions (and without resorting to random oracles). However, they left as an open problem that of designing an encryption scheme that simultaneously provides security against both key dependent chosen plaintext and adaptive chosen ciphertext attack (KDM-CCA2 security). In this paper, we solve this problem. First, we show that by applying the Naor-Yung "double encryption" paradigm, one can combine any KDM-CPA secure scheme with any (ordinary) CCA2 secure scheme, along with an appropriate non-interactive zero-knowledge proof, to obtain a KDM-CCA2 secure scheme. Second, we give a concrete instantiation that makes use the above KDM-CPA secure scheme of BHHO, along with a generalization of the Cramer-Shoup CCA2 secure encryption scheme, and recently developed pairing-based NIZK proof systems. This instantiation increases the complexity of the BHHO scheme by just a small constant factor.
\end{abstract}

\section{Introduction}

Encryption is the oldest cryptographic primitive; indeed, cryptography used to be synonymous with encryption. Despite this, the right definition for the security of encryption schemes has still not been settled! The first formal definition of security for public key encryption was that of semantic security [17], which, loosely speaking, states that given an encryption of a message an adversary cannot learn any information about the message itself. As it turned out, this notion of security does not offer sufficient protection for most practical applications [6], as it does not take into account that an adversary could learn (partial information about) some plaintext when he has access to a decryption oracle. The subsequent stronger notion of security against chosen ciphertext attacks (CCA2 security [31]) takes this into consideration and gives an adversary access to a

The original version of this chapter was revised: The copyright line was incorrect. This has been corrected. The Erratum to this chapter is available at DOI: 10.1007/978-3-642-01001-9_35

A. Joux (Ed.): EUROCRYPT 2009, LNCS 5479, pp. 351-368, 2009.

(C) Springer-Verlag Berlin Heidelberg 2009 
decryption oracle that will decrypt any ciphertext except a particular "challenge ciphertext". CCA2 security was considered the final answer with regard to the security of public key encryption schemes.

However, none of the above notions of security allow an adversary to obtain encryptions of secret keys or, more generally, functions of secret keys. Black, Rogaway, and Shrimpton formally defined such a notion, calling it Key Dependent Message (KDM) security [5]. A similar notion, called circular security, was earlier defined by Camenisch and Lysyanskaya [11] and used to prevent sharing of credentials. Both papers provided constructions in the random oracle model.

Without resorting to the use of random oracles, constructing a public key encryption scheme (practical or not) that is semantically secure against key dependent chosen plaintext attack (KDM-CPA) was a long-standing open problem. It was only recently that Boneh et al. [9] gave a construction of a KDM-CPA secure public key encryption scheme. They proved their scheme secure under the Decisional Diffie-Hellman (DDH) assumption. We will refer to their scheme as the BHHO scheme, which extends to obtain KDM-CPA security under the more general $K$-linear assumption [36,27] (which includes the DDH assumption for $K=1$ and the DLIN assumption [8] for $K=2$ ). However, Boneh et al. left as an open problem the construction of an encryption scheme that is simultaneously secure against key dependent chosen plaintext and chosen ciphertext attack (KDM-CCA2).

Our Contribution. In this paper, we solve this problem by constructing the first KDM-CCA2 secure public key encryption scheme that can be proved secure under standard assumptions, and without random oracles. In fact, we show that a variation of the Naor-Yung paradigm [30] allows one to combine any KDMCPA secure encryption scheme and any regular CCA2 secure encryption scheme, together with a non-interactive zero knowledge (NIZK) proof [7], to obtain a KDM-CCA2 secure encryption scheme.

Moreover, we give a nearly practical instantiation of our general construction using the BBHO KDM-CPA scheme, a $K$-linear version [14,36,23] of the CramerShoup [13] CCA2 scheme, and recently developed pairing-based NIZK proof systems $[19,18,20]$. In the BHHO scheme, a ciphertext is a couple of hundred group elements and our construction blows this up only be a small constant factor (two or three, depending on the cryptographic assumption one employs). For our construction, we need a pairing $e: \mathbb{G} \times \Gamma \rightarrow \mathbb{G}_{\mathrm{T}}$, and we prove security under the $K$-linear assumption in $\mathbb{G}$ and the $L$-linear assumption in $\Gamma$, for appropriate constants $K$ and $L$ (and we also need a collision-resistant hash function).

Motivational Example: Key-Wrap. The "key-wrap" problem motivates the need for KDM-CCA2 secure encryption in practice. The key-wrap mechanism is found, for instance, in cryptographic coprocessors such as IBM's Common Cryptographic Architecture [25] and RSA's Public Key Cryptographic Standards [33]. Cryptographic coprocessors are tamper-proof hardware tokens that process requests from applications to perform cryptographic tasks such as encryption, signing and so on. One can view these tokens as trusted hardware that stores keys of all users in the 
system. When an application (or user) wishes to perform a cryptographic task, it authenticates itself to the token and the token processes the request. For the purpose of creating backup of data or to transport keys from one token to another, it is often desired to encrypt keys (also known as "key wrapping"). Naturally, when we encrypt private keys with other keys it might lead to a circularity. In other words, an adversary might get to see an encryption of a secret key $s k_{1}$ with public key $p k_{2}$ as well as an encryption of a secret key $s k_{2}$ with public key $p k_{1}$ (such circularity can in general be more complicated). Although one can circumvent this problem by maintaining a hierarchy of keys and/or by maintaining separate keys for the purpose of wrapping other keys, this is not always convenient or possible. In addition, since the hardware token performs decryption, an adversary may effectively have access to a decryption oracle.

Labeled Encryption. In many applications in which one uses a CCA2 secure encryption scheme, the notion of a label is very useful. Very briefly, a label consists of public data which is non-malleably attached to a ciphertext. In effect, it allows the encryptor to control the context in which a ciphertext is decrypted. This notion has been around for a long time, under various names, e.g., "indicator", "tag", "header", "associated data" [28,38,37,12,29,26,32]. While one can always implement the label mechanism by appending the label to the plaintext, this is often not the most practical way to achieve this.

Coming back to the key-wrap problem, a label may be used to describe the type of message being encrypted: if it encrypts a key, who the key belongs to, etc. When the hardware token decrypts a ciphertext labeled as a key, it can restrict the usage of the decrypted key; in particular, the token can ensure that such a key is only used within the token in appropriate ways (e.g., decryption, further key-wrap). Even if a token restricts the usage in this way, an adversary may attempt a chosen ciphertext attack by submitting an encryption of a key that actually belongs to Alice, and make it look like it belongs to Bob; moreover, perhaps the adversary is authorized to decrypt ciphertexts under Bob's key, which in effect allows him to decrypt ciphertexts encrypted under Alice's key. However, if labels are used as described above, CCA2 security will prevent such attacks from succeeding.

Because of their utility, we include labels in our definition of KDM-CCA2 security, and implement them in our construction. Moreover, we exploit the label mechanism for plain CCA2 encryption in our general construction to bind together the two ciphertexts and NIZK proof of the Naor-Yung paradigm. In particular, we shall see that the CCA2 encryption scheme we use directly support labels in a way that interacts very nicely with pairing-based NIZK techniques, leading to a conceptually simple and quite efficient concrete instantiation of our general construction.

Another use of labels is to enlarge the message space of a CCA2 encryption scheme: to encrypt a sequence of messages as a package, one can generate a key pair for a strongly secure one-time signature scheme, and then encrypt each message in the sequence using the verification key as a label, and then signing the whole sequence of ciphertexts. This application is convenient for us, because the BHHO scheme can only encrypt one bit of a secret key at a time. 
Other related work. Backes, Pfitzmann and Scedrov [2] and Backes, Dürmuth and Unruh [1] considered KDM-CCA2 security of symmetric and asymmetric encryption schemes, respectively. They in fact define a notion of security stronger than we consider in our paper, by allowing the adversary to obtain some of the secret keys. They showed that RSA-OAEP ([4]) is secure in this sense in the random oracle model.

Halevi and Krawczyk [22] studied key-dependent message security (under the name key-dependent input (KDI) security) with respect to primitives such as pseudo-random functions (PRFs) and block ciphers. They showed that in the idealcipher model, KDI secure PRFs can be built if one restricts the functions of the key to be independent of the ideal-cipher. Further, they showed that this goal cannot be achieved in the standard model. On the positive side, they show that if one allows the PRF construction to depend on a fixed public value, but does not allow the function of the key to depend on this value, then KDI secure PRFs can be constructed in the standard model. Hofheinz and Unruh [24], constructed a symmetric key encryption scheme that achieves KDM-CPA security when an adversary can only make a bounded number of encryptions. Haitner and Holenstein [21] proved negative results for KDM-CPA security of encryption schemes when an adversary can query encryptions of specific functions of the secret key.

Outline of the paper. In $\S 2$, we give and discuss the definitions of KDM-CCA2, NIZK proofs, and strong one-time signatures, i.e., the ingredients of our generic construction, which is presented in $\S 3$.

In $\S 4$, we present concrete instantiations of our building blocks: We recall the BHHO KDM-CPA encryption scheme, the $K$-linear version of the CramerShoup CCA2 encryption scheme, and Groth's strongly secure one-time signature scheme. As a service to the reader, we give a self-contained exposition of a simplified version of the NIZK proof system of Groth and Sahai [20] as it applies to linear equations over a group. This allows us to completely describe the instantiation of our construction and analyze its complexity.

In the full version of the paper [10], we discuss an alternative construction of KDM-CCA2 encryption that uses a CPA secure encryption scheme instead of a CCA2 secure encryption scheme but requires an NIZK proof system that provides (unbounded) simulation soundness [34,35]. In the full paper, we also show how to make the general NIZK proofs of [20] (unbounded) simulation sound, given a CCA2 secure encryption scheme that supports ciphertexts with labels, which again illustrates the power labels.

\section{Preliminaries}

\subsection{Notation}

When we say that an algorithm is efficient, we mean that the algorithm runs in probabilistic polynomial time in the security parameter. All our algorithms and functions take as input an implicit security parameter. When we say that a function is negligible, we mean that it is negligible in the implicit security parameter. Let $a \| b$ denote the concatenation of string $a$ with string $b$. 


\subsection{Definition of KDM-CCA2 Security}

Let $\mathbf{E}$ be a public key encryption system that supports ciphertexts with labels, which consists of three (probabilistic) efficient algorithms EncKeyGen, E and D. EncKeyGen is a randomized key generation algorithm, that outputs a public key/secret key pair $(p k, s k)$. The algorithm $\mathrm{E}$ takes as input a message $m$ (from the message space $\mathcal{M}$ ), a public key $p k$ and a label $\ell$, and outputs a ciphertext $c:=\mathrm{E}(p k, m, \ell)$. When we need to explicitly refer to the randomness in the encryption, we shall refer to an encryption of a message $m$ with randomness $r$ by $\mathrm{E}(p k, m, \ell ; r)$. The decryption algorithm $\mathrm{D}$ takes as input a secret key $s k$, a ciphertext $c$, and a label $\ell$, and either outputs a message $m$ or reject. The (perfect) correctness condition is that (with probability one) $\mathrm{D}(s k, \mathrm{E}(p k, m, \ell), \ell)=m$ for all messages $m$, labels $\ell$ and $(p k, s k)$ pairs output by EncKeyGen.

When we use a public key encryption scheme $\mathbf{E}$ that does not support labels, we refer to the encryption and decryption algorithms of such a scheme by $\mathrm{E}(p k, m)$ and $\mathrm{D}(s k, c)$, respectively.

We extend the definition of key dependent message security from Black et al. [5] to the notion of security against chosen ciphertext attack $([30,31,15])$. We will note that the standard definitions of public key encryption security are specific instances of this definition.

Let $\mathcal{S}$ denote the space of secret keys output by EncKeyGen. As in [22] and [9], key-dependence is defined with respect to a fixed set of functions $\mathcal{C}$. Let $n>0$ be an integer and let $\mathcal{C}$ be a finite set of functions $\mathcal{C}:=\left\{f: \mathcal{S}^{n} \rightarrow \mathcal{M}\right\}$. KDMsecurity is defined with respect to $\mathcal{C}$ through the following two experiments between a challenger and an adversary $\mathcal{A}$. Let $\mathfrak{d} \in \mathcal{M}$ be a fixed (dummy) message in $\mathcal{M}$. Experiment $b$ (where $b=0,1$ ) is defined as follows:

1. Initialization phase: In both experiments the challenger runs EncKeyGen() $n$ times and obtains $n$ key pairs $\left(p k_{1}, s k_{1}\right),\left(p k_{2}, s k_{2}\right), \cdots,\left(p k_{n}, s k_{n}\right)$. It sends the vector $\left(p k_{1}, p k_{2}, \cdots, p k_{n}\right)$ to $\mathcal{A}$.

2. Query phase: In both experiments, $\mathcal{A}$ may adaptively make the following two types of queries to the challenger.

(a) Encryption queries: $\mathcal{A}$ can make a query of the form $(i, f, \ell)$, where $1 \leq i \leq n, f \in \mathcal{C}$ and $\ell$ is a label. The challenger responds by setting $m:=f\left(s k_{1}, s k_{2}, \cdots, s k_{n}\right) \in \mathcal{M}$.

In Experiment $b=0$, it sets $c:=\mathrm{E}\left(p k_{i}, m, \ell\right)$.

In Experiment $b=1$, it sets $c:=\mathrm{E}\left(p k_{i}, \mathfrak{d}, \ell\right)$.

In both experiments, the challenger sends $c$ to $\mathcal{A}$.

When the adversary $\mathcal{A}$ submits $(i, f, \ell)$ as an encryption query and the response of the challenger is $c$, we call $(i, c, \ell)$ a target tuple.

(b) Decryption queries: In both experiments, $\mathcal{A}$ can make a query of the form $(i, c, \ell)$, where $1 \leq i \leq n, c$ is a string to be decrypted using secret key $s k_{i}$ and $\ell$ is a label. The only restriction is that $(i, c, \ell)$ cannot be a (previous) target tuple. Note that $c$ might not necessarily be a valid 
ciphertext. That is, $c$ might not be an output of $\mathrm{E}\left(p k_{j}, m, \ell\right)$ for some $1 \leq j \leq n, m \in \mathcal{M}$ and $\ell$.

In both experiments, the challenger responds with $\mathrm{D}\left(s k_{i}, c, \ell\right)$.

3. Final phase: Finally, the adversary outputs a bit $b^{\prime} \in\{0,1\}$.

Definition 1 (KDM-CCA2). A public key encryption scheme $\mathbf{E}$ is KDMCCA2 secure with respect to $\mathcal{C}$ if $\left|\operatorname{Pr}\left[W_{0}\right]-\operatorname{Pr}\left[W_{1}\right]\right|$ is negligible for all efficient adversaries $\mathcal{A}$, where $W_{b}$ is the event that $\mathcal{A}$ outputs $b^{\prime}=1$ in Experiment $b$.

Note that the standard security definitions for public key encryption can be viewed as specific instances of the above general definition.

KDM-CPA: By restricting $\mathcal{A}$ from making any decryption queries, we get the definition of key-dependent message semantic security (KDM-CPA) as defined in [9].

CCA2: When we restrict the set of functions $\mathcal{C}$ from which $\mathcal{A}$ can draw $f$ to the set of all constant functions on $\mathcal{S}^{n} \rightarrow \mathcal{M}$, we get the experiment for multiple message, multiple key CCA2 security, which is equivalent to the standard CCA2 security for a single message and single key (see [3]). If we further restrict $\mathcal{A}$ from making any decryption queries, we obtain the standard definition for semantic security (also see [3]).

Also note that, unlike regular CPA and CCA2 security, for both KDM-CPA and KDM-CCA2 security, one cannot reduce the attack game to a single encryption query and a single key pair.

We note that the definition of security by Backes et al. [1] is somewhat stronger in that it allows the adversary to obtain some secret keys. To benefit from this in practice, the users need to carefully keep track of which keys were compromised, and which keys are related to each other via key-wrap. In contrast, our definition pessimistically assumes that if one key is compromised then all potentially related keys should be considered compromised as well — which is probably more realistic.

\subsection{Non-interactive Zero-Knowledge Proofs}

Let $R$ be a binary relation that is efficiently computable. For pairs of the form $(x, w) \in R, x$ is referred to as the statement and $w$ as the witness. Let $\mathcal{L}:=\{x$ : $(x, w) \in R$ for some $w\}$.

A non-interactive proof system for $R$ consists of the following efficient algorithms: a common reference string (CRS) generation algorithm CRSGen, a prover $\mathrm{P}$, and a verifier $\mathrm{V}$. The CRSGen algorithm outputs the CRS denoted by $\mathfrak{C}$. $\mathrm{P}$ takes as input $\mathfrak{C}$, statement $x$, and witness $w$. It produces a proof $\mathfrak{p}$ if $(x, w) \in R$ and outputs failure otherwise. $\mathrm{V}$ takes as input $\mathfrak{C}, x$, and $\mathfrak{p}$. $\mathrm{V}$ outputs accept if it accepts the proof and reject otherwise.

Definition 2 (NIZK[7,16]). (CRSGen, P, V) is a non-interactive zeroknowledge (NIZK) proof system for $R$ if it has the following properties described below: 
Perfect Completeness: For all $\mathfrak{C}$ output by CRSGen( $)$, for all $(x, w) \in R$, and for all $\mathfrak{p}:=\mathrm{P}(\mathfrak{C}, x, w), \operatorname{Pr}[\mathrm{V}(\mathfrak{C}, x, \mathfrak{p})$ outputs reject $]=0$.

Computational Soundness: Consider the following game:

1. CRSGen() is run to obtain $\mathfrak{C}$, which is given to the adversary $\mathcal{A}$.

2. $\mathcal{A}$ responds with $(x, \mathfrak{p})$.

$\mathcal{A}$ wins the game if $\mathrm{V}(\mathfrak{C}, x, \mathfrak{p})=$ accept and $x \notin \mathcal{L}$. Let $W$ be the event that $A$ wins the game. Then, for all efficient adversaries $\mathcal{A}$, we have $\operatorname{Pr}[W]$ is negligible.

Computational Zero-knowledge: Let $\mathrm{S}:=\left(\mathrm{S}_{1}, \mathrm{~S}_{2}\right)$ be a simulator running in polynomial time. Consider the following two experiments:

Experiment 0: CRSGen () is run and the output $\mathfrak{C}$ is given to $\mathcal{A}$. $\mathcal{A}$ is then given oracle access to $\mathrm{P}(\mathfrak{C}, \cdot, \cdot)$.

Experiment 1: $\mathrm{S}_{1}()$ generates $\mathfrak{C}$ and trapdoor $\mathfrak{t} . \mathcal{A}$ is given $\mathfrak{C}$, and is then given oracle access to $\mathbf{S}^{\prime}(\mathfrak{C}, \mathfrak{t}, \cdot, \cdot)$, where $\mathbf{S}^{\prime}(\mathfrak{C}, \mathfrak{t}, x, w)$ is defined to be $\mathbf{S}_{2}(\mathfrak{C}, \mathfrak{t}, x)$ if $(x, w) \in R$ and failure if $(x, w) \notin R$.

Let $W_{i}$ be the event that $\mathcal{A}$ outputs 1 in Experiment $i$, for $i=0$ or 1 . Then, for all efficient adversaries $\mathcal{A}$, we have $\left|\operatorname{Pr}\left[W_{0}\right]-\operatorname{Pr}\left[W_{1}\right]\right|$ is negligible.

Note that Blum et al. [7] give a weaker, "one time" definition of computational zero-knowledge, in which the adversary is allowed to see only one fake proof. However, because we cannot reduce KDM-security to an attack game with a single encryption query, this is insufficient for our purposes.

\subsection{Strongly Secure One-Time Signatures}

We also require a strongly secure one-time signature scheme. This is a signature scheme that satisfies the following security property: after obtaining the verification key and a signature $\mathfrak{s}$ on any message $m$ of its choice, it is infeasible for an efficient adversary to generate any valid signature $\mathfrak{s}^{*}$ on any message $m^{*}$ with $\left(\mathfrak{s}^{*}, m^{*}\right) \neq(\mathfrak{s}, m)$. See [10] for a more formal definition.

\section{Generic Construction of a KDM-CCA2 Secure Scheme}

In this section, we give a generic construction of KDM-CCA2 secure public key encryption scheme $\mathbf{E}$ with respect to a set of functions $\mathcal{C}$. We require the following building blocks: a public key encryption scheme $\mathbf{E}_{\mathrm{kdm}}$ that is KDMCPA secure with respect to the set of functions $\mathcal{C}$; a regular CCA2 secure public key encryption scheme $\mathbf{E}_{\text {cca }}$ that supports ciphertexts with labels; an NIZK proof system $\mathbf{P}$ for the language $\mathcal{L}_{\text {eq }}$ consisting of the set of all pairs of ciphertexts that encrypt the same message using $\mathbf{E}_{\mathrm{kdm}}$ and $\mathbf{E}_{\mathrm{cca}}$; and a strongly secure one-time signature scheme $\mathbf{S}$. 
At a high level, $\mathbf{E}$ is similar to the construction of $[30,15]$. To encrypt a message $m$, we generate a key-pair for the scheme $\mathbf{S}$, encrypt $m$ using both $\mathbf{E}_{\mathrm{kdm}}$ and $\mathbf{E}_{\mathrm{cca}}$, where the label for $\mathbf{E}_{\text {cca }}$ will contain the verification key generated above (along with any input label). Using $\mathbf{P}$, we give a proof that both ciphertexts contain the same plaintext. We then sign the two ciphertexts as well as the proof using S. The final ciphertext consists of the verification key, the two ciphertexts, the proof, and the signature.

\subsection{Construction}

We now formally describe the scheme $\mathbf{E}:=$ (EncKeyGen, E, D) in detail. Let $\mathbf{E}_{\mathrm{kdm}}:=\left(\right.$ EncKeyGen $\left._{\mathrm{kdm}}, \mathrm{E}_{\mathrm{kdm}}, \mathrm{D}_{\mathrm{kdm}}\right)$ (with key pair $\left(p k_{\mathrm{kdm}}, s k_{\mathrm{kdm}}\right)$ ) and let $\mathbf{E}_{\text {cca }}:=\left(E_{\text {ncKeyGen }}\right.$ cca $\left., \mathrm{E}_{\text {cca }}, \mathrm{D}_{\text {cca }}\right)$ (with key pair $\left.\left(p k_{\text {cca }}, s k_{\text {cca }}\right)\right)$. Let $\mathbf{S}:=$ (SignKeyGen, Sign, Verify). Let $\mathcal{L}_{\text {eq }}$ be the set of all triples $\left(c_{1}, c_{2}, \ell\right)$ such that

$$
\exists m, r_{1}, r_{2}: c_{1}=\mathrm{E}_{\mathrm{kdm}}\left(p k_{\mathrm{kdm}}, m ; r_{1}\right) \wedge c_{2}=\mathrm{E}_{\mathrm{cca}}\left(p k_{\mathrm{cca}}, m, \ell ; r_{2}\right) .
$$

Let $\mathbf{P}:=($ CRSGen, $\mathrm{P}, \mathrm{V})$ be an NIZK proof system for $\mathcal{L}_{\text {eq }}$. Note that there maybe be common system parameters that are used to define $\mathbf{E}_{\mathrm{kdm}}, \mathbf{E}_{\mathrm{cca}}$, and $\mathbf{P}$, and these are input to all associated algorithms. The encryption scheme $\mathbf{E}$ comprises of the following three algorithms.

\section{EncKeyGen():}

1. Run EncKeyGen kdm () and EncKeyGen eca $_{\text {(n) }}()$ to obtain key pairs $\left(p k_{\mathrm{kdm}}, s k_{\mathrm{kdm}}\right)$ and $\left(p k_{\mathrm{cca}}, s k_{\mathrm{cca}}\right)$, respectively.

2. Run CRSGen() to generate the CRS $\mathfrak{C}$ of the NIZK proof system $\mathbf{P}$.

The public key is $p k:=\left(p k_{\mathrm{kdm}}, p k_{\mathrm{cca}}, \mathfrak{C}\right)$. The secret key is $s k:=s k_{\mathrm{kdm}}$. $\mathrm{E}(p k, m, \ell)$ :

1. Let $c_{\mathrm{kdm}}:=\mathrm{E}_{\mathrm{kdm}}\left(p k_{\mathrm{kdm}}, m ; r_{\mathrm{kdm}}\right)$.

2. Run SignKeyGen() to generate key pair $\left(V K_{\text {ots }}, S K_{\text {ots }}\right)$.

3. Let $c_{\mathrm{cca}}:=\mathrm{E}_{\mathrm{cca}}\left(p k_{\mathrm{cca}}, m, \ell \| V K_{\mathrm{ots}} ; r_{\mathrm{cca}}\right)$.

4. Let $\mathfrak{p}$ be the NIZK proof (using $\mathbf{P})$ for $\left(c_{\mathrm{kdm}}, c_{\text {cca }}, \ell \| V K_{\text {ots }}\right) \in \mathcal{L}_{\text {eq }}$.

5 . Let $c^{\prime}:=c_{\mathrm{kdm}}\left\|c_{\mathrm{cca}}\right\| \mathfrak{p}$ and let $\mathfrak{s}:=\operatorname{Sign}_{S K_{\mathrm{ots}}}\left(c^{\prime}\right)$.

Then $\mathrm{E}(p k, m, \ell):=c_{\mathrm{kdm}}\left\|c_{\mathrm{cca}}\right\| \mathfrak{p}\left\|V K_{\text {ots }}\right\| \mathfrak{s}$.

$\mathrm{D}(s k, c, \ell)$ : Parse $c$ as $c_{\mathrm{kdm}}\left\|c_{\mathrm{cca}}\right\| \mathfrak{p}\left\|V K_{\text {ots }}\right\| \mathfrak{s}$ (and output reject if this fails). Output reject if either $\operatorname{Verify}_{V K_{\mathrm{ots}}}\left(c_{\mathrm{kdm}}\left\|c_{\mathrm{cca}}\right\| \mathfrak{p}, \mathfrak{s}\right)=$ reject or $\mathrm{V}\left(\mathfrak{C},\left(c_{\mathrm{kdm}}, c_{\mathrm{cca}}, \ell \| V K_{\mathrm{ots}}\right), \mathfrak{p}\right)=$ reject; otherwise, output $\mathrm{D}_{\mathrm{kdm}}\left(s k, c_{\mathrm{kdm}}\right)$.

The (perfect) correctness of the public key encryption scheme $\mathbf{E}$ trivially follows from the (perfect) correctness of the scheme $\mathbf{E}_{\mathrm{kdm}}$, (perfect) completeness of the proof system $\mathbf{P}$, and the (perfect) correctness of the signature scheme $\mathbf{S}$.

\subsection{Proof of Security}

Theorem 1. Let $\mathbf{E}_{\mathrm{kdm}}$ be a KDM-CPA secure scheme with respect to the set of functions $\mathcal{C}$. Let $\mathbf{E}_{\mathrm{cca}}$ be a CCA2 secure scheme, $\mathbf{S}$ a strong one-time signature scheme, and $\mathbf{P}$ an NIZK proof system for $\mathcal{L}_{\mathrm{eq}}$. Then $\mathbf{E}$, as constructed above, is a KDM-CCA2 secure scheme with respect to $\mathcal{C}$. 
Proof. The proof is through a sequence of games. We first present a schematic description of the sequence of games used to prove that $\mathbf{E}$ is KDM-CCA2 secure. The underlined parts indicate what has changed in each game.

\begin{tabular}{|c|c|c|c|}
\hline Game & Process encrypt query & Process decrypt query & justification \\
\hline 0 & enc. $(m, m) ;$ real $\mathfrak{p}$ & dec. $c_{\mathrm{kdm}}$ & \\
\hline 1 & enc. $(m, m)$; real $\mathfrak{p}$ & dec. $c_{\mathrm{cca}}$ & soundness for $\mathbf{P}$ \\
\hline 2 & enc. $(m, m)$; fake $\mathfrak{p}$ & dec. $\overline{c_{\mathrm{cca}}}$ & ZK for $\mathbf{P}$ \\
\hline 3 & enc. $(m, m)$; fake $\mathfrak{p}$ & dec. $c_{\text {cca }}$; special reject & strong one-time sig. $\mathbf{S}$ \\
\hline 4 & enc. $(m, \underline{\mathfrak{d}}) ;$ fake $\mathfrak{p}$ & dec. $c_{\text {cca }} ; \overline{\text { special reject }}$ & $\mathrm{CCA} 2$ for $\mathbf{E}_{\mathrm{cca}}$ \\
\hline 5 & enc. $(m, \mathfrak{d}) ;$ fake $\mathfrak{p}$ & dec. $c_{\text {cca }}$; no special reject & strong one-time sig. $\mathbf{S}$ \\
\hline 6 & enc. $(\underline{\mathfrak{d}}, \mathfrak{d}) ; \quad$ fake $\mathfrak{p}$ & dec. $c_{\text {cca }}$ & KDM-CPA for $\mathbf{E}_{\mathrm{kdm}}$ \\
\hline 7 & enc. $(\mathfrak{d}, \mathfrak{d}) ; \quad$ real $\mathfrak{p}$ & dec. $c_{\text {cca }}$ & ZK for $\mathbf{P}$ \\
\hline 8 & enc. $(\mathfrak{d}, \mathfrak{d}) ; \quad$ real $\mathfrak{p}$ & dec. $\underline{c_{\mathrm{kdm}}}$ & soundness for $\mathbf{P}$ \\
\hline
\end{tabular}

The sequence of games involving the challenger $\mathrm{Ch}$ and adversary $\mathcal{A}$ are more formally described below. Let $W_{i}$ be the event that $\mathcal{A}$ outputs 1 in Game $i$.

Game 0: This is the actual attack game, i.e., Experiment 0 in Definition 1.

When responding to an encryption query, Ch encrypts the actual message $m$ using both encryption schemes. The label for $\mathrm{E}_{\text {cca }}$ additionally contains $V K_{\text {ots }}$ which $\mathrm{Ch}$ picks using SignKeyGen(). Ch gives a real proof $\mathfrak{p}$ that both encryptions contain the same message. It produces the signature $\mathfrak{s}$ using $S K_{\text {ots }}$.

Game 1: This game is exactly like Game 0, except that when responding to a decryption query, $C h$ decrypts using secret key $s k_{\text {cca }}$ instead of $s k_{\mathrm{kdm}}$. It follows from the soundness of the proof system $\mathbf{P}$ that $\left|\operatorname{Pr}\left[W_{1}\right]-\operatorname{Pr}\left[W_{0}\right]\right|$ is negligible.

Game 2: This game is exactly like Game 1, except that when responding to an encryption query, $C h$ gives a simulated proof $\mathfrak{p}$ (using the trapdoor of the proof system) instead of a real proof. It follows from the zero-knowledge property of $\mathbf{P}$ that $\left|\operatorname{Pr}\left[W_{2}\right]-\operatorname{Pr}\left[W_{1}\right]\right|$ is negligible.

Game 3: This game is exactly like Game 2, except that when responding to a decryption query of the form $(i, c, \ell)$ from $\mathcal{A}$ such that $c=$ $c_{\mathrm{kdm}}\left\|c_{\text {cca }}\right\| \mathfrak{p}\left\|V K_{\text {ots }}\right\| \mathfrak{s}$, Ch first checks if there exists a target tuple of the form $\left(i, c^{*}, \ell\right)$, with $c^{*}=c_{\mathrm{kdm}}^{*}\left\|c_{\mathrm{cca}}\right\| \mathfrak{p}^{*}\left\|V K_{\mathrm{ots}}\right\| \mathfrak{s}^{*}$ for some $c_{\mathrm{kdm}}^{*}, \mathfrak{p}^{*}$ and $\mathfrak{s}^{*}$. If this is the case, then let $c^{*}$ be the first such response by $\mathrm{Ch}$. Now if $c^{*} \neq c$, then $\mathrm{Ch}$ rejects the encryption query. We call this the special rejection rule. It follows from the strong one-time security of the signature scheme $\mathbf{S}$ that Ch rejects via the special rejection rule only with negligible probability and hence $\left|\operatorname{Pr}\left[W_{3}\right]-\operatorname{Pr}\left[W_{2}\right]\right|$ is negligible.

In Game 3, Ch never decrypts a ciphertext that was contained in a target tuple using $s k_{\text {cca }}$. We can therefore make use of the CCA2 security of $\mathbf{E}_{\text {cca }}$.

Game 4: This game is exactly like Game 3, except that when responding to an encryption query, $C h$ encrypts the dummy message $\mathfrak{d}$ using $E_{\text {cca }}$ but still encrypts the actual message $m$ using $\mathrm{E}_{\mathrm{kdm}}$. It follows from the CCA2 security of $\mathbf{E}_{\text {cca }}$ that $\left|\operatorname{Pr}\left[W_{4}\right]-\operatorname{Pr}\left[W_{3}\right]\right|$ is negligible. 
Game 5: This game is exactly like Game 4, except that when responding to a decryption query, $\mathrm{Ch}$ no longer follows the special rejection rule that was defined in Game 3. It follows from the strong one-time security of the signature scheme $\mathbf{S}$, that $\left|\operatorname{Pr}\left[W_{5}\right]-\operatorname{Pr}\left[W_{4}\right]\right|$ is negligible.

Game 6: This game is exactly like Game 5, except that when responding to an encryption query, Ch encrypts the dummy message $\mathfrak{d}$ using both encryption schemes. It follows from the KDM-CPA security of $\mathbf{E}_{\mathrm{kdm}}$ that $\mid \operatorname{Pr}\left[W_{6}\right]-$ $\operatorname{Pr}\left[W_{5}\right] \mid$ is negligible.

Game 7: This game is exactly like Game 6, except that when responding to an encryption query, $C h$ gives a real proof $\mathfrak{p}$ that both encryptions contain the same message. It follows from the zero-knowledge property of $\mathbf{P}$ that $\left|\operatorname{Pr}\left[W_{7}\right]-\operatorname{Pr}\left[W_{6}\right]\right|$ is negligible.

Game 8: This game is exactly like Game 7, except that when responding to a decryption query, Ch decrypts using secret key $s k_{\mathrm{kdm}}$ instead of $s k_{\text {cca }}$. It follows from the soundness of the proof system $\mathbf{P}$ that $\left|\operatorname{Pr}\left[W_{8}\right]-\operatorname{Pr}\left[W_{7}\right]\right|$ is negligible. Game 8 is Experiment 1 in Definition 1.

Combining the different games, we get that $\left|\operatorname{Pr}\left[W_{8}\right]-\operatorname{Pr}\left[W_{0}\right]\right|$ is negligible, which proves Theorem 1. A more detailed proof can be found in [10].

Note that we used the computational soundness property of the proof system $\mathbf{P}$ only in Games 1 and 8 and in both these games, Ch only gave real proofs for true statements. Hence "plain" soundness of $\mathbf{P}$ is sufficient and we do not require the proof system to be simulation sound ([34]). In the definition of KDM-CCA2 security, one cannot reduce the attack game to a single encryption query and a single public key. Therefore, one-time zero-knowledge (see remark after Definition 2) would not be sufficient for our proof (one-time zero-knowledge does not imply multi-proof zero-knowledge). However, note that CCA2 security is sufficient, as the "single instance" definition implies the "multi-instance" definition (see remark after Definition 1).

\section{Specific Number-Theoretic Instantiation of a KDM-CCA2 Secure Scheme}

In this section, we give specific efficient instantiations of the building blocks used to construct the generic scheme presented in $\S 3$. We introduce notation and the number-theoretic assumptions in $\S 4.1$. In $\S 4.2$, we describe the KDMCPA scheme of Boneh et al. [9], while in $\S 4.3$, we describe the $K$-linear version of the Cramer-Shoup CCA2 encryption scheme that we need. In $\S 4.4$ and $\S 4.5$, we describe the NIZK proof system used to prove equality of plaintexts. We use the efficient strongly one-time signature scheme of Groth [18] (which we describe in $\S 4.6$ ), to complete our instantiation of a KDM-CCA2 secure scheme. In $\S 4.7$, we discuss the size of the public key, system parameters, and ciphertext of our encryption scheme. 


\subsection{General Notation and Assumptions}

Let $\mathbb{G}$ be a group of prime order $q$. We shall write $\mathbb{G}$ using multiplicative notation. One naturally views $\mathbb{G}$ as a vector space over $\mathbb{Z}_{q}$, where for $x \in \mathbb{Z}_{q}$ and $\mathbf{g} \in \mathbb{G}$, the "scalar product" of $x$ and $\mathbf{g}$ is really the power $\mathbf{g}^{x}$. Because of this, we shall often employ concepts and terminology from linear algebra.

For vectors $\overrightarrow{\mathbf{g}}:=\left(\mathbf{g}_{1}, \ldots, \mathbf{g}_{R}\right) \in \mathbb{G}^{R}$ and $\vec{x}:=\left(x_{1}, \ldots, x_{R}\right) \in \mathbb{Z}_{q}^{R}$, define $\langle\overrightarrow{\mathbf{g}}, \vec{x}\rangle:=\mathbf{g}_{1}^{x_{1}} \ldots \cdot \mathbf{g}_{R}^{x_{R}} \in \mathbb{G}$. When we write $\prod_{i=1}^{K} \overrightarrow{\mathbf{g}}_{i} \in \mathbb{G}^{R}$ for vectors $\overrightarrow{\mathbf{g}}_{i} \in \mathbb{G}^{R}$, we mean the component wise product of each of the $R$ terms. Unless otherwise specified, there is no a priori relation between $\mathbf{g}, \overrightarrow{\mathbf{g}}, \mathbf{g}_{i}$ and $\overrightarrow{\mathbf{g}}_{i}$.

Definition 3 ( $K$-linear assumption $[36,27])$. Let $\mathbb{G}$ be a group of prime order $q$. For a constant $K \geq 1$, the $K$-linear assumption in $\mathbb{G}$ is defined through the following two experiments (0 and 1$)$ between a challenger and an adversary $\mathcal{A}$ that outputs 0 or 1 .

Experiment 0: The challenger picks $K+1$ random generators of $\mathbb{G}$ : $\mathbf{g}_{1}, \mathbf{g}_{2}, \ldots, \mathbf{g}_{K+1}$, picks random $x_{1}, \ldots, x_{K} \in \mathbb{Z}_{q}$ and sets $x_{K+1}=\sum_{i=1}^{K} x_{i}$. $\mathcal{A}$ is given $\left(\mathbf{g}_{1}, \mathbf{g}_{2}, \ldots, \mathbf{g}_{K+1}, \mathbf{g}_{1}^{x_{1}}, \mathbf{g}_{2}^{x_{2}}, \ldots, \mathbf{g}_{K+1}^{x_{K+1}}\right)$ as input.

Experiment 1: The challenger picks $K+1$ random generators of $\mathbb{G}$ : $\mathbf{g}_{1}, \mathbf{g}_{2}, \ldots, \mathbf{g}_{K+1}$ and picks random $x_{1}, x_{2}, \ldots, x_{K+1} \in \mathbb{Z}_{q} \cdot \mathcal{A}$ is given $\left(\mathbf{g}_{1}, \mathbf{g}_{2}, \ldots, \mathbf{g}_{K+1}, \mathbf{g}_{1}^{x_{1}}, \mathbf{g}_{2}^{x_{2}}, \ldots, \mathbf{g}_{K+1}^{x_{K+1}}\right)$ as input.

The $K$-linear assumption holds in $\mathbb{G}$ if for all efficient adversaries $\mathcal{A}, \mid \operatorname{Pr}\left[W_{0}\right]$ $\operatorname{Pr}\left[W_{1}\right] \mid$ is negligible, where $W_{i}$ is the event that $\mathcal{A}$ outputs 1 in Experiment $i$.

Another way to understand the $K$-linear assumption is as follows. Let us define group vectors $\overrightarrow{\mathbf{g}}_{1}, \ldots, \overrightarrow{\mathbf{g}}_{K} \in \mathbb{G}^{K+1}: \overrightarrow{\mathbf{g}}_{1}:=\left(\mathbf{g}_{1}, 1,1, \ldots, 1, \mathbf{g}_{K+1}\right), \overrightarrow{\mathbf{g}}_{2}:=$ $\left(1, \mathbf{g}_{2}, 1, \ldots, 1, \mathbf{g}_{K+1}\right), \ldots, \overrightarrow{\mathbf{g}}_{K}:=\left(1,1, \ldots, 1, \mathbf{g}_{K}, \mathbf{g}_{K+1}\right)$. Let $\mathbb{T}$ denote the subspace of $\mathbb{G}^{K+1}$ generated by $\overrightarrow{\mathbf{g}}_{1}, \ldots, \overrightarrow{\mathbf{g}}_{K}$. The $K$-linear assumption says that it is hard to distinguish random elements of $\mathbb{T}$ from random elements of $\mathbb{G}^{K+1}$. Note that the standard Decisional Diffie-Hellman (DDH) assumption is the 1linear assumption and the linear assumption (introduced in [8]) is the 2-linear assumption.

Pairings. Let $\mathbb{G}, \Gamma$ and $\mathbb{G}_{\mathrm{T}}$ be groups of prime order $q$. We shall use Roman letters to denote elements in $\mathbb{G}$ and Greek letters to denote elements in $\Gamma$. A pairing is a map $e: \mathbb{G} \times \Gamma \rightarrow \mathbb{G}_{\mathrm{T}}$ that satisfies the following properties: (1) $e$ is bilinear, which means that for all $\mathbf{a} \in \mathbb{G}$ and $\alpha \in \Gamma$, the maps $e(\mathbf{a}, \cdot): \Gamma \rightarrow \mathbb{G}_{\mathrm{T}}$ and $e(\cdot, \alpha): \mathbb{G} \rightarrow \mathbb{G}_{\mathrm{T}}$ are linear maps; (2) $e$ is non-degenerate, which means that its image is not $\{1\}$; and (3) $e$ is efficiently computable.

\subsection{KDM-CPA Secure Scheme Based on the K-Linear Assumption}

In this section, we describe the public key encryption scheme of Boneh et al. [9] based on the $K$-linear assumption. Let $N:=\left\lceil(K+2) \log _{2} q\right\rceil$. $\mathbf{E}_{\mathrm{kdm}}=$ (EncKeyGen $\mathrm{kdm}_{\mathrm{k}}, \mathrm{E}_{\mathrm{kdm}}, \mathrm{D}_{\mathrm{kdm}}$ ) is as described below. The message space of this scheme is the group $\mathbb{G}$. 
EncKeyGen $_{\mathrm{kdm}}$ :

1. Pick random $\overrightarrow{\mathbf{g}}_{1}, \ldots, \overrightarrow{\mathbf{g}}_{K} \in \mathbb{G}^{N}$.

2. Pick random $\vec{s} \in\{0,1\}^{N}$.

3. Define $\mathbf{h}_{i}:=\left\langle\overrightarrow{\mathbf{g}}_{i}, \vec{s}\right\rangle \in \mathbb{G}$ for $i=1, \ldots, K$.

4. Output the secret key $s k_{\mathrm{kdm}}:=\vec{s}$ and the public key $\mathrm{E}_{\mathrm{kdm}}\left(p k_{\mathrm{kdm}}, \mathbf{m}\right)$ : $p k_{\mathrm{kdm}}:=\left(\overrightarrow{\mathbf{g}}_{1}, \ldots, \overrightarrow{\mathbf{g}}_{K}, \mathbf{h}_{1}, \ldots, \mathbf{h}_{K}\right)$.

1. Pick random $r_{1}, \ldots, r_{K} \in \mathbb{Z}_{q}$.

2. Output the ciphertext $(\mathbf{g}, \mathbf{h}):=\left(\prod_{i=1}^{K} \overrightarrow{\mathbf{g}}_{i}^{r_{i}}, \mathbf{m} \cdot \prod_{i=1}^{K} \mathbf{h}_{i}^{r_{i}}\right) \in \mathbb{G}^{N} \times \mathbb{G}$. $\mathrm{D}_{\mathrm{kdm}}\left(s k_{\mathrm{kdm}},(\overrightarrow{\mathbf{g}}, \mathbf{h})\right)$ : Output $\mathbf{m}:=\mathbf{h} /\langle\overrightarrow{\mathbf{g}}, \vec{s}\rangle$.

Note that the $i^{\text {th }}$ bit $s_{i}$ of the secret key $\vec{s}$ is encoded for the purpose of encryption as $\mathbf{g}^{s_{i}}$ for some random (but fixed) $\mathbf{g} \in \mathbb{G}$.

The key space (of encoded secret keys) is $\mathbb{G}^{N}$. Define a function $f_{\vec{t}, \mathbf{b}}: \mathbb{G}^{n N} \rightarrow$ $\mathbb{G}$ for fixed $\vec{t} \in \mathbb{Z}_{q}^{n N}$ and $\mathbf{b} \in \mathbb{G}$ to be the map $f_{\vec{t}, \mathbf{b}}(\overrightarrow{\mathbf{u}}):=\langle\overrightarrow{\mathbf{u}}, \vec{t}\rangle \cdot \mathbf{b}$. Let $\mathcal{C}$ be the set of all functions $f_{\vec{t}, \mathbf{b}}$ for all values of $\vec{t} \in \mathbb{Z}_{q}^{n N}$ and $\mathbf{b} \in \mathbb{G}$. $\mathbf{E}_{\mathrm{kdm}}$ is KDM-CPA secure with respect to the set of functions $\mathcal{C}[9]$.

Note that [9] explicitly describes the above scheme in the case $K=1$, and only briefly mentions its generalization to $K>1$ (the explicit description of which has been obtained from the authors of [9] via personal communication).

\subsection{CCA2 Secure Scheme Based on the $K$-Linear Assumption}

In this section, we describe a generalized version of the Cramer-Shoup encryption scheme based on the $K$-linear assumption. This generalization was described in [23] and [36]. However, given the $K$-linear decision problem, this scheme is essentially already implicit in [14] (based on Theorems 2 and 3, along with Example 1 in $\S 7.4$, of the full length version of that paper). This scheme is CCA2 secure and supports ciphertexts with labels. $\mathbf{E}_{\text {cca }}=\left(E_{n c K e y G e n}{ }_{c c a}, E_{c c a}, D_{\text {cca }}\right)$ is as described below. The message space of this scheme is the group $\mathbb{G}$, and the label space is $\{0,1\}^{*}$.

EncKeyGen $_{\text {cca }}$ :

1. Pick random $\mathbf{f}_{1}, \ldots, \mathbf{f}_{K+1} \in \mathbb{G}$.

2. Pick random $\vec{x}, \vec{y}, \vec{z} \in \mathbb{Z}_{q}^{K+1}$.

3. Define the following elements of $\mathbb{G}^{K+1}: \overrightarrow{\mathbf{f}}_{1}:=\left(\mathbf{f}_{1}, 1,1, \ldots, 1, \mathbf{f}_{K+1}\right), \overrightarrow{\mathbf{f}}_{2}:=$ $\left(1, \mathbf{f}_{2}, 1, \ldots, 1, \mathbf{f}_{K+1}\right), \ldots, \overrightarrow{\mathbf{f}}_{K}:=\left(1,1, \ldots, 1, \mathbf{f}_{K}, \mathbf{f}_{K+1}\right)$.

4. Define the following elements of $\mathbb{G}: \mathbf{c}_{i}:=\left\langle\overrightarrow{\mathbf{f}}_{i}, \vec{x}\right\rangle, \mathbf{d}_{i}:=\left\langle\overrightarrow{\mathbf{f}}_{i}, \vec{y}\right\rangle, \mathbf{e}_{i}:=$ $\left\langle\overrightarrow{\mathbf{f}}_{i}, \vec{z}\right\rangle \quad(i=1, \ldots, K)$.

5 . Output the secret key $s k_{\text {cca }}:=(\vec{x}, \vec{y}, \vec{z})$ and the public key $p k_{\text {cca }}:=$ $\left(\left\{\mathbf{f}_{j}\right\}_{j=1}^{K+1},\left\{\mathbf{c}_{i}\right\}_{i=1}^{K},\left\{\mathbf{d}_{i}\right\}_{i=1}^{K},\left\{\mathbf{e}_{i}\right\}_{i=1}^{K}\right)$.

$\mathrm{E}_{\text {cca }}\left(p k_{\text {cca }}, \mathbf{m}, \ell\right)$ :

1. Pick random $w_{1}, \ldots, w_{K} \in \mathbb{Z}_{q}$. 
2. Compute $(\overrightarrow{\mathbf{f}}, \mathbf{a}, \mathbf{b}):=\left(\prod_{i=1}^{K} \overrightarrow{\mathbf{f}}_{i}^{w_{i}}, \mathbf{m} \cdot \prod_{i=1}^{K} \mathbf{c}_{i}^{w_{i}}, \prod_{i=1}^{K}\left(\mathbf{d}_{i} \mathbf{e}_{i}^{t}\right)^{w_{i}}\right) \in \mathbb{G}^{K+1} \times$ $\mathbb{G} \times \mathbb{G}$, where $t:=H(\overrightarrow{\mathbf{f}}, \mathbf{a}, \ell) \in \mathbb{Z}_{q}$ and $H$ is a collision resistant hash function. Output the ciphertext is $(\overrightarrow{\mathbf{f}}, \mathbf{a}, \mathbf{b})$.

$\mathrm{D}_{\text {cca }}\left(s k_{\text {cca }},(\overrightarrow{\mathbf{f}}, \mathbf{a}, \mathbf{b}), \ell\right)$ :

1. Verify that $\mathbf{b}=\langle\overrightarrow{\mathbf{f}}, \vec{y}+t \vec{z}\rangle$.

2. Output $\mathbf{m}:=\overrightarrow{\mathbf{a}} /\langle\overrightarrow{\mathbf{f}}, \vec{x}\rangle$.

Note that the schemes in $[14,36,23]$ do not explicitly support labels; however, the proof of security immediately generalizes to allow this, provided one assumes (as we do) that $H$ is collision resistant.

\subsection{NIZK Proofs for Satisfiable Systems of Linear Equations over Groups}

In this section, we describe the NIZK proofs for proving that a system of linear equations over a group is satisfiable. These proofs are derived from Groth and Sahai [20]. The paper [20] deals with much more general systems of equations; for many applications, such as ours, we only need linear equations. For completeness, and concreteness, we describe how the methods of [20] apply to this setting. Our exposition is self contained, but brief.

Let $\mathbb{G}$ be a group of prime order $q$. A linear equation over $\mathbb{G}$ is an equation of the form $\mathbf{g}_{0}=\prod_{j=1}^{W} \mathbf{g}_{j}^{\mathbf{x}_{j}}$, where $\mathbf{g}_{0}, \mathbf{g}_{1}, \ldots, \mathbf{g}_{W} \in \mathbb{G}$ are constants and $\mathrm{x}_{1}, \ldots, \mathrm{X}_{W}$ are variables. An assignment to the variables is a tuple $\left(x_{1}, \ldots, x_{W}\right) \in \mathbb{Z}_{q}^{W}$, and such an assignment satisfies the equation if $\mathbf{g}_{0}=\prod_{j=1}^{W} \mathbf{g}_{j}^{x_{j}}$. A set $S$ of linear equations over $\mathbb{G}$ is called satisfiable if there exists an assignment to the variables that simultaneously satisfies each equation in $S$.

Let $\mathcal{L}_{\text {lsat }}$ be the language of all satisfiable sets of linear equations over $\mathbb{G}$. A witness for membership in $\mathcal{L}_{\text {lsat }}$ is a satisfying assignment. Our goal is to construct an efficient NIZK proof system for $\mathcal{L}_{\text {lsat }}$.

Our proof system for $\mathcal{L}_{\text {lsat }}$ requires a pairing $e: \mathbb{G} \times \Gamma \rightarrow \mathbb{G}_{\mathrm{T}}$, where $\Gamma$ and $\mathbb{G}_{\mathrm{T}}$ are also groups of order $q$. In addition, we need to make the $L$-linear assumption in $\Gamma$, for some constant $L$ (typically, $L$ is a small constant like 1 or 2 , depending on the assumption we make).

- The CRS generator works as follows:

1. Pick random $\gamma_{1}, \ldots, \gamma_{L+1} \in \Gamma$.

2. Define the following elements of $\Gamma^{L+1}: \vec{\gamma}_{1}:=\left(\gamma_{1}, 1, \ldots, 1, \gamma_{L+1}\right), \vec{\gamma}_{2}:=$ $\left(1, \gamma_{2}, \ldots, 1, \gamma_{L+1}\right), \ldots, \vec{\gamma}_{L}:=\left(1,1, \ldots, \gamma_{L}, \gamma_{L+1}\right)$.

3. Choose $\vec{\gamma} \in \Gamma^{L+1}$ at random.

4. The common reference string is $\left(\gamma_{1}, \ldots, \gamma_{L+1}, \vec{\gamma}\right)$.

- Given a set $S$ of equations, along with a satisfying assignment $\left(x_{1}, \ldots, x_{W}\right)$, the prover works as follows:

1. Commit to $x_{1}, \ldots, x_{W}$ by setting $\vec{\delta}_{j}:=\vec{\gamma}^{x_{j}} \prod_{k=1}^{L} \vec{\gamma}_{k}^{r_{j k}}$, for $j=1, \ldots, W$, where the $r_{j k}$ 's are randomly chosen elements of $\mathbb{Z}_{q}$. 
2. The proof consists of the commitments $\vec{\delta}_{1}, \ldots, \vec{\delta}_{W}$, and, in addition, for each equation $\mathbf{g}_{0}=\prod_{j=1}^{W} \mathbf{g}_{j}^{\mathbf{x}_{j}}$ in $S$, the proof contains $L$ corresponding "proof elements" $\mathbf{p}_{1}, \ldots, \mathbf{p}_{L} \in \mathbb{G}$, which are computed as: $\mathbf{p}_{k}:=\prod_{j=1}^{W} \mathbf{g}_{j}^{r_{j k}}(k=1, \ldots, L)$.

- To verify such a proof, the verifier takes the commitments $\vec{\delta}_{1}, \ldots, \vec{\delta}_{W}$, and, for each equation $\mathbf{g}_{0}=\prod_{j=1}^{W} \mathbf{g}_{j}^{\mathbf{x}_{j}}$ in $S$, takes the corresponding proof elements $\mathbf{p}_{1}, \ldots, \mathbf{p}_{L}$, and checks that

$$
\prod_{j=1}^{W} E\left(\mathbf{g}_{j}, \vec{\delta}_{j}\right)=E\left(\mathbf{g}_{0}, \vec{\gamma}\right) \prod_{k=1}^{L} E\left(\mathbf{p}_{k}, \vec{\gamma}_{k}\right) .
$$

Here, $E: \mathbb{G} \times \Gamma^{L+1} \rightarrow \mathbb{G}_{\mathrm{T}}^{L+1}$ is the biliear map that sends $\left(\mathbf{g},\left(\alpha_{1}, \ldots, \alpha_{L+1}\right)\right)$ to $\left(e\left(\mathbf{g}, \alpha_{1}\right), \ldots, e\left(\mathbf{g}, \alpha_{L+1}\right)\right)$.

The CRS contains $2(L+1)$ elements of $\Gamma$, and a proof consists of $W(L+1)$ elements of $\Gamma$ (for the commitments) and $|S| L$ elements of $\mathbb{G}$ (for the proof elements).

We now show that the above proof system has perfect completeness, (statistical) soundness, and computational zero-knowledge.

Perfect completeness. To argue perfect completeness, using bilinearity, one checks by a simple calculation that for any satisfying assignment $\left(x_{1}, \ldots, x_{W}\right)$, and for any choice of the $r_{j k}$ 's, equation (1) will always be satisfied.

Soundness. A simple fact that will be useful in proving both the soundness and zero-knowledge property is the following, which the reader can easily verify using bilinearity:

Lemma 1. If $\vec{\beta}_{1}, \ldots, \vec{\beta}_{R} \in \Gamma^{L+1}$ are linearly independent, then the map

$$
\left(\mathbf{h}_{1}, \ldots, \mathbf{h}_{R}\right) \mapsto E\left(\mathbf{h}_{1}, \vec{\beta}_{1}\right) \cdots E\left(\mathbf{h}_{R}, \vec{\beta}_{R}\right)
$$

is an injective linear map from $\mathbb{G}^{R}$ into $\mathbb{G}_{\mathrm{T}}^{L+1}$.

To prove soundness, note that with overwhelming probability, the vectors $\vec{\gamma}, \vec{\gamma}_{1}, \ldots, \vec{\gamma}_{L}$ form a basis for $\Gamma^{L+1}$. Suppose a proof contains commitments $\vec{\delta}_{1}, \ldots, \vec{\delta}_{W} \in \Gamma^{L+1}$. Regardless of how these commitments were actually computed, each $\vec{\delta}_{j}$ can be expressed uniquely as $\vec{\delta}_{j}=\vec{\gamma}^{x_{j}} \prod_{k=1}^{L} \vec{\gamma}_{k}^{r_{j k}}$ for some $x_{j}, r_{j 1}, \ldots, r_{j L} \in \mathbb{Z}_{q}$. Now consider any particular equation $\mathbf{g}_{0}^{*}=\prod_{j=1}^{W} \mathbf{g}_{j}^{\mathbf{x}_{j}}$, and corresponding proof elements $\mathbf{p}_{1}^{*}, \ldots, \mathbf{p}_{L}^{*}$. Define $\mathbf{g}_{0}:=\prod_{j=1}^{W} \mathbf{g}_{j}^{x_{j}}$ and $\mathbf{p}_{k}:=\prod_{j=1}^{W} \mathbf{g}_{j}^{r_{j k}}$ for $k=1, \ldots, L$, using the $x_{j}$ 's and $r_{j k}$ 's determined as above by the commitments. On the one hand, by perfect completeness, we have $\prod_{j=1}^{W} E\left(\mathbf{g}_{j}, \vec{\delta}_{j}\right)=E\left(\mathbf{g}_{0}, \vec{\gamma}\right) \prod_{k=1}^{L} E\left(\mathbf{p}_{k}, \vec{\gamma}_{k}\right)$. On the other hand, if the verification equation (1) holds for the given equation and proof elements, then we also must have $\prod_{j=1}^{W} E\left(\mathbf{g}_{j}, \vec{\delta}_{j}\right)=E\left(\mathbf{g}_{0}^{*}, \vec{\gamma}\right) \prod_{k=1}^{L} E\left(\mathbf{p}_{k}^{*}, \vec{\gamma}_{k}\right)$. Thus, we have $E\left(\mathbf{g}_{0}, \vec{\gamma}\right) \prod_{k=1}^{L} E\left(\mathbf{p}_{k}, \vec{\gamma}_{k}\right)=E\left(\mathbf{g}_{0}^{*}, \vec{\gamma}\right) \prod_{k=1}^{L} E\left(\mathbf{p}_{k}^{*}, \vec{\gamma}_{k}\right)$. Applying Lemma 1 to the linearly independent vectors $\vec{\gamma}, \vec{\gamma}_{1}, \ldots, \vec{\gamma}_{L}$, we conclude that $\mathbf{g}_{0}=\mathbf{g}_{0}^{*}$ (and in fact, $\mathbf{p}_{k}=\mathbf{p}_{k}^{*}$ for $\left.k=1, \ldots, L\right)$. It follows that if the proof verifies, then the assignment $x_{1}, \ldots, x_{W}$ determined by the commitments simultaneously satisfies all the given equations. 
Zero Knowledge. The simulator generates a "fake CRS" as follows: it generates $\vec{\gamma}_{1}, \ldots, \vec{\gamma}_{L}$ as usual, but it computes $\vec{\gamma}$ as $\prod_{k=1}^{L} \vec{\gamma}_{j}^{s_{j}}$ for random $s_{1}, \ldots, s_{L} \in$ $\mathbb{Z}_{q}$. The trapdoor for the fake CRS is $\left(s_{1}, \ldots, s_{L}\right)$.

In a fake CRS, $\vec{\gamma}_{1}, \ldots, \vec{\gamma}_{L}$ are linearly independent (with overwhelming probability), while $\vec{\gamma}$ is a random element of the subspace $V$ generated by $\vec{\gamma}_{1}, \ldots, \vec{\gamma}_{L}$.

To simulate a proof for a satisfiable set $S$ of linear equations, the simulator starts by setting $\vec{\delta}_{j}:=\prod_{k=1}^{L} \vec{\gamma}_{k}^{r_{j k}}$ for random $r_{j k} \in \mathbb{Z}_{q}$ for $j=1, \ldots, W$ and $k=1, \ldots, L$. For each equation $\mathbf{g}_{0}=\prod_{j=1}^{W} \mathbf{g}_{j}^{\mathbf{x}_{j}}$ in $S$, the simulator generates proof elements $\mathbf{p}_{1}, \ldots, \mathbf{p}_{L}$ as follows: $\mathbf{p}_{k}:=\mathbf{g}_{0}^{-s_{k}} \prod_{j=1}^{W} \mathbf{g}_{j}^{r_{j k}} \quad(k=1, \ldots, L)$. The reader may easily verify, using the bilinearity property, that the verification equation (1) is satisfied.

We now argue that fake proofs are computationally indistinguishable from real proofs. To this end, let us introduce a hybrid prover, which works exactly like a real prover, except that it uses a fake CRS. Such hybrid proofs are computationally indistinguishable from real proofs, under the $L$-linear assumption for $\Gamma$. Moreover, hybrid proofs are statistically indistinguishable from fake proofs. To see this, observe that with overwhelming probability, $\vec{\gamma}_{1}, \ldots, \vec{\gamma}_{L}$ are linearly independent. Assuming this is true, in both the hybrid and fake proofs, the distribution of the commitments are the same (uniformly and independently distributed over the subspace $V$ ). Additionally, in both types of proofs, the proof elements $\mathbf{p}_{1}, \ldots, \mathbf{p}_{L}$ for a given equation are uniquely determined in the same way by the equation, the commitments, and the CRS; indeed, both types of provers generate proof elements that satisfy the verification equation (1); moreover, applying Lemma 1 to the vectors $\vec{\gamma}_{1}, \ldots, \vec{\gamma}_{L}$, we see that for a fixed equation, commitments, and CRS, there exist unique $\mathbf{p}_{1}, \ldots, \mathbf{p}_{L}$ that satisfy (1).

\subsection{NIZK Proof for Proving Equality of Plaintext}

Given a ciphertext of $\mathbf{E}_{\mathrm{kdm}}($ from $\S 4.2)$ of the form $(\overrightarrow{\mathbf{g}}, \mathbf{h}) \in \mathbb{G}^{N} \times \mathbb{G}$ and a ciphertext of $\mathbf{E}_{\text {cca }}($ from $\S 4.3)$ of the form $(\overrightarrow{\mathbf{f}}, \mathbf{a}, \mathbf{b}) \in \mathbb{G}^{K+1} \times \mathbb{G} \times \mathbb{G}$ with respect to a label $\ell \in\{0,1\}^{*}$, we want to prove that they are valid encryptions of the same message. This is done by proving that there exist $r_{1}, \ldots, r_{K}, w_{1}, \ldots, w_{K} \in$ $\mathbb{Z}_{q}$ such that $\overrightarrow{\mathbf{g}}=\prod_{i=1}^{K} \overrightarrow{\mathbf{g}}_{i}^{r_{i}}, \overrightarrow{\mathbf{f}}=\prod_{i=1}^{K} \overrightarrow{\mathbf{f}}_{i}^{w_{i}}, \mathbf{b}=\prod_{i=1}^{K}\left(\mathbf{d}_{i} \mathbf{e}_{i}^{t}\right)^{w_{i}}$, and $\mathbf{h} / \mathbf{a}=$ $\prod_{i=1}^{K} \mathbf{h}_{i}^{r_{i}} / \prod_{i=1}^{K} \mathbf{c}_{i}^{w_{i}}$, where $t:=H(\overrightarrow{\mathbf{f}}, \mathbf{a}, \ell)$.

This translates into $N+(K+1)+1+1=N+K+3$ equations in $2 K$ variables. Using the proof system above, this means we need $(2 K)(L+1)$ elements of $\Gamma$ for commitments, and $(N+K+3) L$ elements of $\mathbb{G}$ for the proofs.

\subsection{Strongly Secure One-Time Signature Scheme}

Here is the strongly secure one-time signature scheme $\mathbf{S}$ from Groth [18]. It makes use of a group $\mathbb{G}$ of prime order $q$ with generator $\mathbf{g}$, and a hash function $H:\{0,1\}^{*} \rightarrow \mathbb{Z}_{q}$. The scheme is secure assuming the hardness of computing discrete logs in $\mathbb{G}$ (which follows from the $K$-linear assumption) and assuming $H$ is collision resistant. 
SignKeyGen: Pick random $x, y \in \mathbb{Z}_{q}^{*}$ and $r, s \in \mathbb{Z}_{q}$, and set $\mathbf{f}:=\mathbf{g}^{x}, \mathbf{h}:=\mathbf{g}^{y}$, and $\mathbf{c}:=\mathbf{f}^{r} \mathbf{h}^{s}$. The verification key is $V K=(\mathbf{f}, \mathbf{h}, \mathbf{c})$ and the secret key $S K=(x, y, r, s)$.

$\operatorname{Sign}_{S K}(m)$ : To sign a message $m \in\{0,1\}^{*}$, pick $t$ at random from $\mathbb{Z}_{q}$. The signature is $\mathfrak{s}=(t,(x(r-t)+y s-H(m)) / y)$.

$\operatorname{Verify}_{V K}(m, \mathfrak{s}):$ To verify the signature $\mathfrak{s}=(t, w)$, check that $\mathbf{c}=\mathbf{g}^{H(m)} \mathbf{f}^{t} \mathbf{h}^{w}$.

\subsection{Size of Public Key, System Parameters and Ciphertext}

Using the $K$-linear assumption for $\mathbb{G}$ and the $L$-linear assumption for $\Gamma$, the size of the public key, system parameters and ciphertext are as follows, where $N:=\left\lceil(K+2) \log _{2} q\right\rceil$.

The system parameters consists of the CRS which comprises $2(L+1)$ elements of $\Gamma$, the descriptions of $\mathbb{G}, \Gamma, \mathbb{G}_{\mathrm{T}}, e$ and the collision resistant hash function $H$ for $\mathbf{E}_{\mathrm{cca}}$ and $\mathbf{S}$.

The public key of $\mathbf{E}$ consists of $(N+1) K$ elements of $\mathbb{G}$ for the public key $p k_{\mathrm{kdm}}$ and $4 K+1$ elements of $\mathbb{G}$ for the public key $p k_{\text {cca }}$, for a total of $(N+5) K+1$ elements of $\mathbb{G}$.

The two ciphertexts $\left(c_{\mathrm{kdm}}\right.$ and $\left.c_{\mathrm{cca}}\right)$ require $(N+1)$ and $(K+3)$ elements of $\mathbb{G}$, respectively, giving a total of $N+K+4$ elements of $\mathbb{G}$. To prove equality of plaintexts, we require $(2 K)(L+1)$ elements of $\Gamma$ for commitments, and $(N+$ $K+3) L$ elements of $\mathbb{G}$ for the proofs. Finally, to sign the resulting ciphertexts and proofs using the one-time signature scheme $\mathbf{S}$, we require 3 elements of $\mathbb{G}$ for the verification key $V K$ of $\mathbf{S}$ and 2 elements of $\mathbb{Z}_{q}$ for the signature.

Note that we can make the public key shorter, by making $p k_{\text {cca }}$ as part of the system parameters; indeed, since the secret key $s k_{\text {cca }}$ is not needed (other than in the proof of security), one can simply generate all of the group elements appearing in $p k_{\mathrm{cca}}$ at random (yielding a distribution that is statistically close to the real distribution on public keys).

We emphasize that, typically, one would set $K=1,2$ and $L=1,2$, depending on the groups $\mathbb{G}$ and $\Gamma$. For example, at one extreme, if $\mathbb{G}=\Gamma$, then one could set $K=L=2$; at the other extreme, if $\mathbb{G} \neq \Gamma$, and there is no (known) efficiently computable homomorphism from $\mathbb{G}$ to $\Gamma$ or vice versa, then one could set $K=L=1$.

\section{References}

1. Backes, M., Dürmuth, M., Unruh, D.: OAEP is secure under key-dependent messages. In: Pieprzyk, J. (ed.) ASIACRYPT 2008. LNCS, vol. 5350, pp. 506-523. Springer, Heidelberg (2008)

2. Backes, M., Pfitzmann, B., Scedrov, A.: Key-dependent message security under active attacks - BRSIM/UC-soundness of symbolic encryption with key cycles. In: CSF, pp. 112-124 (2007)

3. Bellare, M., Boldyreva, A., Micali, S.: Public-key encryption in a multi-user setting: Security proofs and improvements. In: Preneel, B. (ed.) EUROCRYPT 2000. LNCS, vol. 1807, pp. 259-274. Springer, Heidelberg (2000) 
4. Bellare, M., Rogaway, P.: Optimal asymmetric encryption. In: De Santis, A. (ed.) EUROCRYPT 1994. LNCS, vol. 950, pp. 92-111. Springer, Heidelberg (1995)

5. Black, J., Rogaway, P., Shrimpton, T.: Encryption-scheme security in the presence of key-dependent messages. In: Selected Areas in Cryptography, pp. 62-75 (2002)

6. Bleichenbacher, D.: Chosen ciphertext attacks against protocols based on the RSA encryption standard PKCS \#1. In: Krawczyk, H. (ed.) CRYPTO 1998. LNCS, vol. 1462, pp. 1-12. Springer, Heidelberg (1998)

7. Blum, M., Feldman, P., Micali, S.: Non-interactive zero-knowledge and its applications (extended abstract). In: STOC 1988, pp. 103-112 (1988)

8. Boneh, D., Boyen, X., Shacham, H.: Short group signatures. In: Franklin, M. (ed.) CRYPTO 2004. LNCS, vol. 3152, pp. 41-55. Springer, Heidelberg (2004)

9. Boneh, D., Halevi, S., Hamburg, M., Ostrovsky, R.: Circular-secure encryption from decision diffie-hellman. In: Wagner, D. (ed.) CRYPTO 2008. LNCS, vol. 5157, pp. 108-125. Springer, Heidelberg (2008)

10. Camenisch, J., Chandran, N., Shoup, V.: A public key encryption scheme secure against key dependent chosen plaintext and adaptive chosen ciphertext attacks. Cryptology ePrint Archive, Report 2008/375 (2008), http://eprint.iacr.org/

11. Camenisch, J.L., Lysyanskaya, A.: An efficient system for non-transferable anonymous credentials with optional anonymity revocation. In: Pfitzmann, B. (ed.) EUROCRYPT 2001. LNCS, vol. 2045, pp. 93-118. Springer, Heidelberg (2001)

12. Camenisch, J.L., Shoup, V.: Practical verifiable encryption and decryption of discrete logarithms. In: Boneh, D. (ed.) CRYPTO 2003. LNCS, vol. 2729, pp. 126-144. Springer, Heidelberg (2003)

13. Cramer, R., Shoup, V.: A practical public key cryptosystem provably secure against adaptive chosen ciphertext attack. In: Krawczyk, H. (ed.) CRYPTO 1998. LNCS, vol. 1462, pp. 13-25. Springer, Heidelberg (1998)

14. Cramer, R., Shoup, V.: Universal hash proofs and a paradigm for adaptive chosen ciphertext secure public-key encryption. In: Knudsen, L.R. (ed.) EUROCRYPT 2002. LNCS, vol. 2332, p. 45. Springer, Heidelberg (2002), http://eprint.iacr.org/2001/085

15. Dolev, D., Dwork, C., Naor, M.: Non-malleable cryptography (extended abstract). In: STOC 1991, pp. 542-552 (1991)

16. Feige, U., Lapidot, D., Shamir, A.: Multiple non-interactive zero knowledge proofs based on a single random string (extended abstract). In: FOCS 1990, pp. 308-317 (1990)

17. Goldwasser, S., Micali, S.: Probabilistic encryption and how to play mental poker keeping secret all partial information. In: STOC 1982, pp. 365-377 (1982)

18. Groth, J.: Simulation-sound NIZK proofs for a practical language and constant size group signatures. In: Lai, X., Chen, K. (eds.) ASIACRYPT 2006. LNCS, vol. 4284, pp. 444-459. Springer, Heidelberg (2006)

19. Groth, J., Ostrovsky, R., Sahai, A.: Perfect non-interactive zero knowledge for NP. In: Vaudenay, S. (ed.) EUROCRYPT 2006. LNCS, vol. 4004, pp. 339-358. Springer, Heidelberg (2006)

20. Groth, J., Sahai, A.: Efficient non-interactive proof systems for bilinear groups. In: Smart, N.P. (ed.) EUROCRYPT 2008. LNCS, vol. 4965, pp. 415-432. Springer, Heidelberg (2008)

21. Haitner, I., Holenstein, T.: On the (im)possibility of key dependent encryption. In: TCC 2009 (2009)

22. Halevi, S., Krawczyk, H.: Security under key-dependent inputs. In: CCS 2007: Proceedings of the 14th ACM conference on Computer and communications security, pp. 466-475. ACM, New York (2007) 
23. Hofheinz, D., Kiltz, E.: Secure hybrid encryption from weakened key encapsulation. In: Menezes, A. (ed.) CRYPTO 2007. LNCS, vol. 4622, pp. 553-571. Springer, Heidelberg (2007)

24. Hofheinz, D., Unruh, D.: Towards key-dependent message security in the standard model. In: Smart, N.P. (ed.) EUROCRYPT 2008. LNCS, vol. 4965, pp. 108 126. Springer, Heidelberg (2008)

25. IBM. IBM CCA Basic Services Reference and Guide for the IBM 4758 PCI and IBM 4764 PCI-X Cryptographic Coprocessors: Releases 2.53, 2.54, 3.20, 3.23, 3.24, $3.25,3.27$, and $3.30(2008)$

26. Kiltz, E.: Chosen-ciphertext security from tag-based encryption. In: Halevi, S., Rabin, T. (eds.) TCC 2006. LNCS, vol. 3876, pp. 581-600. Springer, Heidelberg (2006)

27. Kiltz, E.: Chosen-ciphertext secure key-encapsulation based on gap hashed diffiehellman. In: Okamoto, T., Wang, X. (eds.) PKC 2007. LNCS, vol. 4450, pp. 282 297. Springer, Heidelberg (2007)

28. Lim, C.H., Lee, P.J.: Another method for attaining security against adaptively chosen ciphertext attacks. In: Stinson, D.R. (ed.) CRYPTO 1993. LNCS, vol. 773, pp. 420-434. Springer, Heidelberg (1994)

29. MacKenzie, P.D., Reiter, M.K., Yang, K.: Alternatives to non-malleability: Definitions, constructions, and applications. In: Naor, M. (ed.) TCC 2004. LNCS, vol. 2951, pp. 171-190. Springer, Heidelberg (2004)

30. Naor, M., Yung, M.: Public-key cryptosystems provably secure against chosen ciphertext attacks. In: STOC 1990, pp. 427-437 (1990)

31. Rackoff, C., Simon, D.R.: Non-interactive zero-knowledge proof of knowledge and chosen ciphertext attack. In: Feigenbaum, J. (ed.) CRYPTO 1991. LNCS, vol. 576, pp. 433-444. Springer, Heidelberg (1992)

32. Rogaway, P., Shrimpton, T.: A provable-security treatment of the key-wrap problem. In: Vaudenay, S. (ed.) EUROCRYPT 2006. LNCS, vol. 4004, pp. 373-390. Springer, Heidelberg (2006)

33. RSA Laboratories. PKCS \#11 v2.20: Cryptographic Token Interface Standard (2004)

34. Sahai, A.: Non-malleable non-interactive zero knowledge and adaptive chosenciphertext security. In: FOCS 1999, pp. 543-553 (1999)

35. De Santis, A., Di Crescenzo, G., Ostrovsky, R., Persiano, G., Sahai, A.: Robust noninteractive zero knowledge. In: Kilian, J. (ed.) CRYPTO 2001. LNCS, vol. 2139, pp. 566-598. Springer, Heidelberg (2001)

36. Shacham, H.: A Cramer-Shoup encryption scheme from the linear assumption and from progressively weaker linear variants. Cryptology ePrint Archive, Report 2007/074 (2007), http://eprint.iacr.org/

37. Shoup, V.: A proposal for an ISO standard for public key encryption, version 2.1 (2001), http://shoup.net/papers/

38. Shoup, V., Gennaro, R.: Securing threshold cryptosystems against chosen ciphertext attack. In: Nyberg, K. (ed.) EUROCRYPT 1998. LNCS, vol. 1403, pp. 1-16. Springer, Heidelberg (1998) 